\title{
Korruptionsentstehung und Korruptionsbekämpfung aus der Unternehmensperspektive
}

\author{
Dipl. Soz.-Oek. Ingo Techmeier
}

\section{Einleitung}

Mit schöner Regelmäßigkeit können die Medien das öffentliche Interesse an Korruptionsvorfällen bedienen. Der Stoff hierfür stammt in der Regel aus öffentlichen Einrichtungen wie zuletzt im Oktober 2004 als Schmiergeldzahlungen aus dem Verkehrsministerium bekannt wurden. Eine mögliche Erklärung für diese Konzentration auf öffentliche Einrichtungen bieten US-amerikanische Daten, aus denen hervorgeht, dass strafrechtliche Ermittlungen bei Betrugsdelikten deutlich wahrscheinlicher sind, wenn eine öffentliche Einrichtung geschädigt wurde (Alexander 1999: 510ff). Daraus sollte mithin nicht der Schluss gezogen werden, dass Korruption in den Geschäftsbeziehungen zwischen Unternehmen keine Rolle spielte. Lediglich der Umgang mit entdeckten Fällen ist ein anderer. Wurden in der Neuen Kriminalpolitik im Sonderheft Wirtschaftskriminalität und Strafverfahren (Heft 4, 2005) verschiedene juristische Perspektiven auf das Phänomen Wirtschaftskriminalität dargestellt, sind die folgenden Ausführungen dem unternehmerischen Blickwinkel auf das Phänomen Korruption und dem Umgang mit ihr gewidmet.

\section{Zum Vorgehen und der Methode}

n Anlehnung an Williamson wird
das Unternehmen als ein Beherr-
schungs- und Überwachungssys-
tem beschrieben und diese Auffas-
sung dahin gehend fortgeführt, dass
aus ihr die Schließung des Unterneh-
mens gegenüber Dritten begründet
wird. Aus dieser Abgrenzung nach
Außen folgt, dass ein Blick ins Innere
eines Unternehmens bspw. durch
die Strafverfolgungsbehörden nach
Möglichkeit vermieden wird. Diese
Annahme wird anhand von qualita-
tiven Experteninterviews empirisch
belegt, die aus einem Forschungs-
projekt zur Wirtschaftskriminalität
im Zusammenhang mit der Privati-
sierung der DDR-Betriebe durch die
Treuhandanstalt stammen. ${ }^{1}$ Damit
wird hier auf dasselbe empirische
Material zurückgegriffen, aus dem
auch Theile (2005) in dem bereits ge-
nannten Sonderheft einige prägnan-
te Passagen zitierte.

Die Interviews dieses Forschungsprojekts wurden anhand eines Leitfadens strukturiert, der einerseits sicher stellen sollte, dass alle uns theoretisch wesentlich erscheinenden Komplexe angesprochen wurden, andererseits aber so offen gestaltet, dass die Gesprächspartner weitere bedeutsame Aspekte einbringen konnten. Diese Konzeption lehnte sich an die von Glaser und Strauss entwickelte Grounded Theory an (vgl. Flick, von Kardorff und Steinke 2000). Aus den mehr als 75 geführten Interviews mit Personen, die an den Privatisierungen oder an der Aufarbeitung strafrechtlicher Vorkommnisse im Zusammenhang mit dem Verkauf der Treuhandbetriebe beteiligt waren, werden einige besonders prägnante Passagen aus den Interviews mit Unternehmensvertretern und später verurteilten Privatisierern zitiert. $^{2}$ Die empirischen Befunde sollen helfen, sich ein Bild von einem abstrakten Unternehmen zu machen, ohne eine Typologisierung von Tätertypen anzustreben. Dies mag man unter Präventionsgesichtspunkten bedauerlich finden, doch selbst da, wo das größte Interesse an einer erfolgreichen Typologisierung angenommen werden kann, bei der Auswahl von Mitarbeitern für ein Unternehmen, ist eine solche nur begrenzt möglich. Entsprechende Versuche stehen vor dem Problem, dass die von einem Manager geforderten Eigenschaften sich sowohl für legale wie für illegale Geschäfte eignen, so Bussmann (2004: 40). Diesem Problem sah sich auch Bannenberg gegenüber, die versuchte Täterprofi- le zu bestimmen. Es gelang ihr zwar, einen Typus „Betrügerpersönlichkeit" ausfindig zu machen, doch handelt es sich hier um einen „eher seltenen Typ" (Bannenberg 2002: 170), während die „meisten Täter (...) keine Auffälligkeiten im Lebensstil (zeigten)" (Bannenberg 2002: 219) Stattdessen muss auch sie konstatieren, dass sich die Täter oftmals durch Eigenschafen auszeichnen, die im Beruf als wünschenswert gelten wie ehrgeizig, engagiert und durchsetzungsstark oder Eigenschaften aufwiesen, die auch unter Normtreuen als verbreitet angenommen werden können wie penibel, übergenau und herrisch (Bannenberg 2002: 219). Versuche, einen zu Delinquenz neigenden Persönlichkeitstyp zu bestimmen, sind daher an sich „wenig ratsam"(Bussmann 2004: 40). ${ }^{3}$

\section{Ein Unternehmen ist ein Beherrschungs- und Über- wachungssystem}

Üblicherweise wird das Unternehmen über seinen Unternehmenszweck definiert und ist in einer zweckorientierten Betrachtung eine Einheit, die das Ziel der Gewinnmaximierung verfolgt. Im Folgendem soll ein anderer Aspekt hervorgehoben werden, der darauf zielt, wie das Unternehmen von dem Markt, auf dem es agiert, unterschieden werden kann. Dieser Unterschied ist ebenso schlicht wie aufschlussreich und stellt darauf ab, wie eine Allokation von beliebigen Ressourcen seien es Arbeitskräfte oder Arbeitsmaterialien - erreicht wird. Auf dem Markt geschieht dies mittels der sofortigen oder auch kreditierten Übergabe von Geld, dagegen erfolgt die Übergabe innerhalb eines Unternehmens aufgrund einer Anordnung (Coase 1988: 34f). So wird ein auf dem Markt für ein Unternehmen erworbener Computer unternehmensintern dem Angestellten zugewiesen, der damit arbeiten soll. Daher muss ein Unternehmen so organisiert sein, dass die notwendigen Anweisungen möglich sind. Erreicht wird dies dadurch, dass die entscheidende Instanz Kenntnisse über vorhandene oder fehlende Arbeitsgeräte hat und in der Position ist, einen Einkauf zu delegieren. Das Thema dieses Aufsatzes erinnert auch den Gutgläubigen daran, dass die Delegation eines Kaufes im doppelten Sinne auf Kosten des delegierenden Unternehmens gehen kann. Dann nämlich, wenn der beauftragte Einkäufer sich beispielsweise nicht von Kriterien wie Preis und Leistung, sondern von einer erhaltenen Bestechungszahlung leiten lässt. Auch ohne die Möglichkeit strafrechtlich relevanter Vorgänge setzt eine auf Anordnung aufbauende Zuteilung von Arbeitsmitteln eine Beobachtung des Innenlebens eines Unternehmens voraus, um überhaupt die notwendige Entscheidungsgrundlage zu haben. Aus diesem Grund kann ein Unternehmen als ein Beherrschungs- und Überwachungssystem aufgefasst werden (Williamson 1990: 14).

Am Markt endet die Herrschaft des Unternehmens

Das Unternehmen wurde als ein System dargestellt, welches intern eine Ressourcenzuteilung durch eine Anordnung vornimmt. Diese Form der Zuteilung endet für das Unternehmen auf dem Markt, da es auf diesem keinen Ressourcentransfer anordnen kann. Stattdessen wird der Transfer hier durch die Zahlung und den Empfang von Geld gesteuert. Um davon nicht zuviel zu bezahlen (oder zu wenig beim Verkauf zu er- 
langen), müssen die Preise verglichen werden, was dann schwierig wird, wenn mit den Preisen auch die Qualität der Produkte variiert.

Es ist offensichtlich, dass ein solcher Vergleich, nur mit einigem Aufwand zu realisieren ist, da in der Regel immer zwei Parameter berücksichtigt werden müssen: Der Preis und die Qualität. Der erste der beiden Parameter ist simpel, da ein Marktteilnehmer wenig zahlen, aber viel einnehmen will. Dagegen ist der zweite sehr komplex, da die Qualität mit dem Preis korreliert ist, kann das entscheidende Kriterium nicht die höchste Qualität sein. Die Aufwendungen, die für eine Beschaffung am Markt getrieben werden müssen, werden in der Ökonomik unter dem Begriff der Transaktionskosten zusammengefasst und bezeichnet die Kosten für die Vertragsanbahnung, die Vertragserfüllung und die Vertragsüberwachung (Coase 1990: 6). Bereits die Vertragsanbahnung ist sehr aufwendig wie das Beispiel der Beschaffung eines Computers für ein Unternehmen zeigt: Es muss ein Modell ausgewählt werden, das den geforderten Ansprüchen genügt, und ein Händler, der unter Berücksichtigung des angebotenen Services zu dem günstigsten Preis anbietet. Des Weiteren ist bei Lieferung die Vertragserfüllung des Verkäufers zu prüfen und, falls Gewährleistungsansprüche in Anspruch genommen werden, ist hierfür die Arbeitszeit eines Angestellten aufzuwenden. Schließlich fallen Kosten an, um sicherzustellen, dass die Angestellten immer im Interesse des Unternehmens handeln. Es sind diese Transaktionskosten, deren Höhe darüber entscheidet, welche Güter und Dienstleistungen innerhalb eines Unternehmens selbst erbracht und welche zugekauft werden (Coase 1988: 7; Williamson 1990: 105). Über die Frage, Zukauf oder Eigenproduktion, entscheidet neben einer Kostenabwägung auch die Gewohnheit - „das wurde schon immer so gemacht" -, deren Überwindung in den Bereich der Innovation fällt. Doch ist die Innovation ebenso wie ihre Generalisierung auf das Kostenkalkül zurückzuführen.

\section{Korruption: Für und Wider das Unternehmen}

Unter Korruption soll schlicht die Übergabe/der Empfang von Geld oder eines geldwerten Vorteils verstanden werden, den jemand dafür erhält, dass er zugunsten des Bestechenden gegen die Interessen seines eigentlichen Auftraggebers handelt: Der Einkäufer des Computers wählt einen bestimmten Computer nicht nach den sachlichen Kriterien von Preis und Qualität aus, sondern nach persönlichen, in dem er den Anbieter bevorzugt, zu dem eine korruptive Beziehung besteht. Aus Unternehmensperspektive sind zwei Arten der Korruption zu unterscheiden: Korruptive Handlungen, die dem Unternehmen nutzen, und solche, die ihm schaden.

Nicht jede Handlung, die der Gewinnmaximierung dient, ist auch im Interesse des Unternehmens. In einem Unternehmen sind nur solche korruptiven Handlungen im Unternehmensinteresse, die angeordnet wurden. Juristisch ist diese Unterscheidung heikel, da strafbewehrtes Tun aus gutem Grund nicht unbedingt so angeordnet wird, dass es von Dritten nachvollziehbar ist. Doch lässt sich der Zweck einer korruptiven Handlung mit der Beschreibung des Unternehmens als Beherrschungssystem fassen: Erwünschte Korruption ist eine Ausdehnung der internen Anordnung in ein fremdes Unternehmen, oder einen Bereich der von Dritten beherrscht wird (beispielsweise in eine Baubehörde), in dem die Korruption den Preisbildungsmechanismus auf dem Markt ersetzt.

Korruptive Handlungen gegen das Interesse des Unternehmens, das den Bestechenden beschäftigt, sind nach der obigen Abgrenzung solche, die das Unternehmen schädigen. Eine solche Schädigung kann in zwei Kategorien eingeteilt werden. In solche, die direkt das Unternehmen schädigen, wenn die eigenen Angestellten aufgrund einer Schmiergeldzahlung unternehmensfremde Interessen vertreten, und solche, die indirekt das Unternehmen schädigen, wenn angeordnete Standards wie beispielsweise die Anordnung, es dürfen keine Bestechungsgelder gezahlt werden, nicht eingehalten werden. Erhöht ein
Verkäufer das Auftragsvolumen mit korruptiven Praktiken, erhöht er damit absolut auch den Gewinn des Unternehmens, gefährdet dabei jedoch gleichzeitig den guten Ruf der begünstigten Firma. Die Entscheidung, was höher zu bewerten ist, der Gewinn oder das Risiko die Reputation zu schädigen, lässt sich die Geschäftsführung nicht aus der Hand nehmen.

\section{Der Umgang mit entdeckter Korruption}

Es macht natürlich einen Unterschied, ob eine strafbewehrte Handlung, die gegen das definierte Unternehmensinteresse verstößt, von Dritten oder von eigenen Angestellten begangen wird. Ebenso, ob delinquente Angestellte (als Bestochene) nur den eigenen Vorteil verfolgten oder (als Bestechende) dem Ziel der Gewinnmaximierung des Unternehmens dienen. Trotz dieser Unterschiede ist die Reaktion des geschädigten Unternehmens soweit sie sich aus den von uns geführten Interviews erschließt - in aller Regel gleich: Es wird eine Lösung ohne Rückgriff auf das Strafrecht gesucht.

Einige Zitate aus den von uns geführten Interviews sollen verdeutlichen, dass das Strafrecht aus unternehmerischer Perspektive auch dann gescheut wird, wenn das eigene Unternehmen Opfer einer strafbewehrten Handlung geworden ist:

Auf die Frage, wie mit einem Angestellten umgegangen werden solle, der sich eines strafrechtsrelevanten Verhalten schuldig gemacht hat, entgegnet ein von uns interviewter Beschuldigter, „eine Kündigung, meine ich, müsste sein. Ob ich ihn jetzt anzeige, ich weiß nicht, ob ich Kraft des Gesetzes (dazu) verpflichtet wäre", (5-4-2; 2736-2848. Hervorhebung durch den Autor.).

In einem anderen Interview mit einem Geschäftsführer (5-13-1; 1922-2262) eines privatisierten Unternehmens ohne strafrechtliche Relevanz wird deutlicher, dass es keine legalistische, sondern eine unternehmerische Entscheidung ist, ob auf das Strafrecht zurückgegriffen wird: „(W)enn einer wirklich echte Straftaten begeht (...), dann muss man sich natürlich sehr schnell entscheiden: Wie geht man nach Außen? Geht man nicht nach Außen? Kann man es noch intern abwickeln oder nicht?"

Auch der Manager eines weiteren Unternehmens, das ohne delinquente Vorkommnisse einen Betrieb der ehemaligen DDR erworben hatte, reagiert auf die Frage, ob das Strafrecht eine Regelungsressource des Unternehmens sei, spontan abwehrend: „Hoffentlich nicht! (...) Erst in letzter Instanz, wenn die Dimension so gewaltig wird, dass man sagt: ,Selbstverständlich müssen wir den zur Rechenschaft ziehen,'" (5-14-1; 44834522).

Interessanterweise haben auch solche Interviewpartner, die nicht als Beschuldigte mit dem Strafrecht in Berührung kamen, eine deutliche Scheu, auf strafrechtliche Regulierungsmöglichkeiten zurückzugreifen. Dafür können zwei Gründe angeführt werden:

Zum einen geht es darum, sich an ein im Geschäftsleben übliches Verfahren zu halten, das als Kompromissfindung bezeichnet werden kann: „Sie können jeden Streit beilegen, wenn sie Anwälte und Wirtschaftsprüfer heraushalten. Die verstehen einfach nicht, dass man im Geschäftsleben Kompromisse machen muss," zitiert Williamson (1990: 12) aus einer US-amerikanischen Studie. Wird dagegen ein Geschäftspartner per Anzeige einem Strafverfahren ausgesetzt, wird die Möglichkeit eines Kompromisses aus der Hand gegeben. Dies kann nicht nur zukünftige Vertragsabschlüsse mit dieser Partei ausschließen, sondern auch weitere potenzielle Geschäftspartner werden sich möglicherweise dem Risiko, im Konfliktfall den Strafverfolgungsbehörden gegenüberzustehen, nicht aussetzen wollen.

Eine externe strafrechtliche Lösung verbindet notwendig den $\mathrm{Na}$ men des eigenen Unternehmens mit dem Strafrecht, da sie mit einer - wenn auch ungewollten - Nähe zu einem Delinquenten verbunden ist. Ein Unternehmen wird nach Möglichkeit immer auf Distanz zu Kriminellen gehen (Kahan und Posner: 1999: 370), stößt es dagegen selber strafrechtliche Ermittlungen an, wird öffentlich, dass es in sei- 
nem Geschäftsumfeld zu strafbaren Handlungen gekommen ist. Auch die Möglichkeit, Dritte könnten vermuten, die Strafverfolgungsbehörden seien aus opportunistischen Gründen eingeschaltet worden, beispielsweise um einem Konkurrenten $\mathrm{zu}$ schaden, entwertet eine strafrechtliche Lösung unter Geschäftsleuten (Kahan und Posner: 1999: 374)

Aus diesem Grund wird eine unternehmensinterne Lösung vorgezogen. Die möglichen Konsequenzen für abweichendes Verhalten, die einem unserer Probanden zuerst einfielen, sind daher solche die ohne Rückgriff auf Dritte auskommen: Es sind die Abmahnung und die Kündigung, (5-13-1; 19222262). Beide Sanktionsmittel erlauben es dem Unternehmen, die Außenwelt aus dem Unternehmen herauszuhalten. Die Einschaltung der Justiz - und damit der Öffentlichkeit im Wege einer öffentlichen Verhandlung - spielt für die Konfliktregulierung keine Rolle, da die stigmatisierende Wirkung des Strafrechts zu groß ist, als dass es ein akzeptiertes Instrument zur Beilegung von Konflikten zwischen Unternehmen sein könnte. Das Ausmaß des strafrechtlichen Stigmas führt dazu, dass sanktionierte Akteure aus höheren Hierarchieebenen ihre Funktion verlieren oder gar vollständig von ihrem bisherigen Tätigkeitsbereich ausgeschlossen werden (Boers, Karliczek und Theile 2003: 486f.).

\section{Die Organisation der Beherr- schung und Überwachung}

Anhand des Prinzipal-AgentenTheorems (Dietz 1998: 29) werden nun die Schwierigkeiten der unternehmensinternen Kontrollen beschrieben. Der Prinzipal ist die Instanz, die das Unternehmensinteresse definiert, üblicherweise die Geschäftsführung, und der Agent ist ein Angestellter, der mit der Umsetzung dieses Interesses betraut wurde. Dass im Falle einer Aktiengesellschaft die Geschäftsführung selber ein von den Aktionären eingesetzter Agent ist, deren Summe wiederum dessen Prinzipal bildet, kann hier vernachlässigt werden. In der folgenden Betrachtung wird ausschließlich das Kontrollverhältnis zwischen Geschäftsführung und ihrem Agenten angesprochen.

Das Verhältnis zwischen dem Prinzipal und dem Agenten ist ein Vertragsverhältnis, so dass auch hier alle bereits angesprochenen Probleme der Vertragsanbahnung, -abwicklung und -überwachung anfallen, die mit Transaktionskosten verbunden sind. Einer vollständigen Überwachung des Agenten könnte man sich durch eine hochgradige Standardisierung seiner Arbeitsabläufe annähern. Die Kontrolle erstreckt sich auch auf die Feststellung möglicher Abweichungen von den festgelegten Standards.

Realiter wäre ein solches Vorgehen mit verschiedenen Problemen behaftet, von denen eines sich daraus ergibt, dass die Kontrolle von ausformulierten Normen wiederum ein eigenes Vertragswerk mit den entsprechenden Transaktionskosten erfordert. Die Folge ist ein infiniter Regress, den der Prinzipal nur dadurch beenden kann, dass er die Überwachung selbst übernimmt. Doch erfasst eine Vertragslösung, welche die Beauftragung eines Agenten immer ist, die Chancen und Risiken einer Delegation immer nur unvollständig, da die Berücksichtigung sämtlicher Handlungsmöglichkeiten unmöglich ist. Oft wird dies mit prohibitiv hohen Kosten begründet (Dietz 1998: 30; Battigalli, Maggi 2002: 799). Doch wichtiger ist, dass Verträge aufgrund der begrenzten Rationalität der Akteure immer unvollständig sind (Williamson 1996: 7; Richter 2001: 226). Auch eine mögliche Annäherung an einen idealen vollständigen Vertrag beseitigte die Probleme des Prinzipals nicht, wenn der Agent zwischen widersprüchlichen Zielen abwägen und das Optimum seiner Tätigkeit aus seiner gegebenen Situation heraus mitbestimmen muss.

Eine Standardisierung, die eine Kontrolle - sei es vollständig oder in Stichproben - des Agenten erst erlaubt, kann unter diesen Bedingungen Aufwendungen erfordern, deren Kosten nicht im Verhältnis zum Ergebnis stehen (Battigalli und Maggi: 2002: 799). Salopp formuliert: Kontrolle muss man sich leisten können. Ein gravierender Nachteil ist die mit einer zunehmenden Standardisierung verbundene Sen- kung der möglichen Flexibilität des Agenten. Je stärker er sich an Vorgaben halten soll, um so mechanischer muss er auf eine sich wandelnde Umwelt reagieren. Individuelle Vertragsanpassungen und -aushandlungen werden dem Agenten so unmöglich gemacht. Mit einer vollständigen Standardisierung wäre der Prinzipal ebenso davor geschützt, dass der Agent seine Position ausnutzt, wie er vor den Vorteilen einer technischen oder anderen Neuerungen geschützt ist. Strenge Standards, die den Agenten auf einen ausgetretenen Pfad zwingen, verhindern erwünschte wie nicht erwünschte Abweichungen.

\section{Die Organisation in Abhängig- keit von der Marktstruktur}

Je stärker ein Produkt bereits durch die übliche Art und Weise, in der es gehandelt wird, normiert ist, desto geringer sind die Möglichkeiten des Agenten, durch korruptive Handlungen entgegen den Interessen seines Prinzipalen, einen Dritten (in ökonomischer Terminologie: Klienten) zu bevorzugen (Dietz 1998: 29). Welchen Aufwand der Prinzipal treiben muss, um durch Standards und deren Kontrolle sich vor korruptiven Handlungen zu schützen, ist dem Teilmarkt geschuldet, auf dem sein Agent agiert.

Ein Markt auf dem eine hohe Standardisierung erreicht ist, ist die Börse (Coase 1988: 8f). Es kann nicht behauptet werden, dass dadurch strafbewehrte Handlungen ausgeschlossen sind, aber in einigen Bereichen sind sie tatsächlich unmöglich. So sind bspw. die gehandelten Produkte, die Aktien eines gegebenen Unternehmens, bei gleichem Nennwert absolut identisch und bieten keine Möglichkeit sich durch Manipulationen der Produktqualität $\mathrm{zu}$ bereichern. Insgesamt erreicht die Börse eine relativ hohe Transparenz, die alle die Aufwendungen, die oben als Transaktionskosten definiert wurden, reduziert.

An einer Börsen werden neben Aktien auch normierte Agrarprodukte und Rohstoffe gehandelt, doch soll die Aktie als Illustration dienen: Die Besonderheit des Aktienmarktes ist, dass auf ihm die Transaktionskosten für Käufer wie für Verkäufer auf ein Mindestmaß gesenkt sind. Hat sich der Käufer einmal entschieden, von welchem Unternehmen er eine Aktie erwerben möchte (um hier zu einer Entscheidung $\mathrm{zu}$ kommen, können beträchtliche Aufwendungen anfallen), dann sieht er sich einer unbestimmten Zahl von Anbietern gegenüber die alle ein identisches Produkt zum gleichen Preis anbieten. Haftungs- und Gewährleistungsansprüche, Kundenservice und ähnliche Überlegungen spielen keine Rolle, darüber hinaus ist die Börsenaufsicht für die Einhaltung der vereinbarten Standards zuständig. Diese hohe Transparenz des Börsenhandels schränkt Freiräume, die zu Lasten Dritter genutzt werden können, erheblich ein.

Ist die Entscheidung getroffen worden, wann und welche Aktien erworben werden sollen, dann herrschen vergleichsweise ideale Bedingungen für den Prinzipalen, der nun von seinem Agenten nichts (oder nicht mehr viel) zu fürchten hat. Solche Bedingungen sind aber üblicherweise nicht gegeben. Die folgenden Überlegungen sind den üblicherweise anzutreffenden Umständen gewidmet.

Bedingungen wie sie am eben genannten Beispiel der Börse herausgearbeitet wurden, sind am ehesten dort zu finden, wo aufgrund einer hohen Standardisierung eine Kontrolle der handelnden Personen vergleichsweise leicht zu bewerkstelligen ist. So wurden anlässlich einer Anti-Korruptions-Kampagne in $\mathrm{Ar}$ gentinien die Einkaufspreise verschiedener Krankenhäuser in $\mathrm{Bu}$ enos Aires miteinander verglichen. Da es sich bei den betrachteten Produkten um einfache Grundsubstanzen wie Ethylalkohol und Wasserstoffperoxyd handelte, waren Preisvariationen nicht mit Qualitätsunterschieden $\mathrm{zu}$ erklären. Unterschiede in den Beschaffungspreisen konnten zwar auch einer fehlenden Motivation des Einkäufers geschuldet sein, jedoch war ein Korruptionsdelikt wahrscheinlicher. Durch eine verstärkte Überwachung der Einkäufer konnten im Anschluss die Beschaffungspreise vorübergehend um $15 \%$ und langfristig um $10 \%$ gesenkt werden (di Tella und Schargrodsky 2003: 270f). 
Bei hochkomplexen Gütern, die sich weitgehend einer Standardisierung entziehen, sind die Kontrollprobleme nur noch unter großen Mühen in den Griff zu bekommen, da es nur schwer möglich ist, eine Grundlage zur Beobachtung des Agenten zu schaffen. Denn dieser muss beim Handel mit komplexen Gütern wegen der zu regelnden Spezifikationen sowie den Gewährleistungen und den Serviceanforderungen oft auch komplexe Kaufverträge für seinen Prinzipal verhandeln und abschließen. Diese mit der Komplexität der gehandelten Güter wachsenden Kontrollprobleme werden als ein Grund dafür genannt, dass auch arme Länder militärische Hochtechnologie importieren (Frank 2004: 187).

\section{Organisierte Flexibilität und widersprüchliche Zielvorgaben}

Korruptive Gelegenheiten ergeben sich nicht nur aus dem Teilmarkt, sondern auch aus den Zielvorgaben des Prinzipalen. Sind diese widersprüchlich, ergeben sich Gelegenheiten, die Interessen Dritter gegenüber denen des Auftraggebers zu bevorzugen. Diese Konsequenz lässt sich anhand der korruptiven Vorgänge um die regionalen Niederlassungen der Treuhandanstalt bei der Privatisierung ehemals volkseigener Betriebe der DDR gut illustrieren.

Die politisch gewünschte schnelle Privatisierung der ehemaligen DDRBetriebe geriet in Gegensatz zu dem Erfordernis, ein effektives Beherrschungs- und Überwachungssystem innerhalb der Treuhansanstalt zu errichten, welches eine effektive Kontrolle der Niederlassungen ermöglichte hätte. Unter anderem fehlte hierfür schlicht die Grundlage beispielsweise bekannte Marktwerte, der $\mathrm{zu}$ privatisierenden Betriebe. Selbst eine Orientierung an Buchwerten war nicht möglich, da die Umstellung der Jahresabschlüsse auf die Normen der alten Bundesländer sich stark verzögerte. So sei ein später verurteilter Privatisierer, 4-21(2), zu Beginn seiner Tätigkeit von seiner Niederlassung in Halle zu einer "Strafpredigt" nach Berlin zum Hauptsitz der Treuhandanstalt berufen worden, weil seine Niederlassung die geringsten Verkaufszahlen gehabt hätte. Er habe dazu erklärt, dass dies an einem Mangel der vorliegenden Bilanzen liege, worauf ihm entgegnet worden sei, dass könne so nicht bleiben, der politische Auftrag hieße, verkaufen um jeden Preis. Die Grenze, die dabei nicht überschritten werden dürfe, sei die Veruntreuung, wenn er dazu nicht in der Lage wäre, würde jemand anderes eingesetzt werden (Privatisierer, 4-2-1(2); 334/356). Das Privatisierungstempo wurde daraufhin von unserem Gesprächspartner ebenso gesteigert wie die Grenze zur Untreue überschritten wurde.

Es kann nun spekuliert werden, ob strafbewehrte Vorgänge im Interesse einer schnellen Privatisierung von politischer Seite billigend in Kauf genommen worden sind, aber das führt vom Thema dieses Artikels weg. Das Beispiel sollte verdeutlichen, dass einem Agenten möglicherweise zwei Ziele vorgegeben werden, die zugleich gar nicht erreicht werden können. Entweder wird ein möglichst schneller Verkauf oder eine juristisch einwandfreie Verkaufsabwicklung (alternativ können hier weitere Zielgrößen genommen werden wie möglichst hoher Verkaufserlös oder die Zukunftsfähigkeit der privatisierten Betriebe) angestrebt. Sind die Ziele widersprüchlich, muss der Agent sich entscheiden, welchem er Vorrang einräumt. Bei seinen Entscheidungsproblemen kann er sich von Dritten mittels Schmiergeldern oder geldwerten Vorteilen „helfen“ lassen.

\section{Schlussbemerkung}

Das Unternehmen wurde als ein Beherrschungs- und Überwachungssystem dargestellt innerhalb dessen Transaktionskosten optimiert werden. In Verfolgung dieser Optimierungsaufgabe muss entschieden werden, welche Leistungen intern im Unternehmen hergestellt und welche extern am Markt zugekauft werden sollen. Während bei der externen Beschaffung ein Teil der Kontrollaufwendungen direkt am Markt erfolgt, muss für die interne Abwicklung ein System installiert sein, dass es ermöglicht, dass Anordnungen sowohl gegeben als auch ausgeführt werden. Dies erfordert eine Optimierung der Aufwen- dungen, um den internen Kontrollanspruch umsetzen zu können und damit eine Steigerung der Transaktionskosten.

Das Ziel dieses internen Anspruchs ist es, den eigenen Kontrollbereich nach Außen vor der Ausdehnung des Kontrollbereichs Dritter zu schützen. Ein solcher Eingriff Dritter wäre beispielsweise ein bestochener Agent im eigenen Unternehmen. Die eigenen Agenten dürfen nur dann auf korruptiven Wege das Unternehmensziel der Profitmaximierung verfolgen, wenn dies - in welcher Form auch immer - angeordnet wird. Ist dagegen eine strafbewehrte Gewinnerhöhung von der Geschäftsführung ausgeschlossen worden, ist die juristische Normtreue der Agenten obligatorisch. Der Prinzipal muss die Bedingungen für die Kontrolle seiner Agenten schaffen. Der Weg hierzu sind überprüfbare Standards, deren Einhaltung kontrollierbar ist. Welcher Aufwand seitens des Unternehmens hierfür auch unter Berücksichtigung der anfallenden Kosten optimal ist, muss innerhalb des Unternehmens abgewogen werden. Es ist wichtig, sich zu vergegenwärtigen, dass für die Schaffung von Standards und ihrer Kontrolle erhebliche Mittel aufgewandt werden müssen, um gegen eine mögliche korruptive Handlung vorgehen beziehungsweise diese präventiv verhindern zu können. Da diese Aufwendungen gleichermaßen für mögliche wie für tatsächliche Handlungen anfallen, werden auch Agenten überwacht, die normtreu sind.

Damit ist jedoch lediglich die Kostenseite der Kontrolle zusammengefasst. Doch ist in vielen Fällen bedeutsamer, dass eine die Überwachung ermöglichende Standardisierung die Flexibilität des Agenten einschränkt. Dies ist bei einfachen Gütern kein Problem, so kann ein Einkäufer, der nach Handelsklassen systematisierte Agrarprodukte in festgesetzten Mengen kauft, tatsächlich ausschließlich nach dem Preis vorgehen und entsprechend einfach, kann seine Tätigkeit kontrolliert werden. Mit steigender Komplexität der zu handelnden Güter wächst jedoch die Anforderung an eine flexible Verhandlungsführung auf beiden Seiten der ver- handelnden Parteien, was eine Kontrolle der jeweils agierenden Agenten erheblich erschwert.

Die vielleicht wichtigste Erkenntnis - vor allem für Interessierte, die mit Korruptionsfällen aus der Perspektive der Strafverfolgungsbehörden zu tun haben - ist, dass Unternehmen in der Regel nicht auf das Strafrecht zurückgreifen, wenn sie Opfer von korruptiven Handlungen werden. Dies begründet eine geringe Anzeigenbereitschaft und korrespondiert mit der Einschätzung des Bundeskriminalamts, dass allgemein Wirtschaftsdelikte „Überwachungs- und Kontrolldelikte" sind (Bundeskriminalamt, 2001, S. 8) und macht die Ahndung von Korruptionsdelikten oder beispielsweise der Untreue nach § 266 (StGB) im Geschäftsverkehr im wesentlichen $\mathrm{zu}$ einem reinen Kontrolldelikt. Eine juristische Kontrolle wird durch den Umstand erschwert, dass das was innerhalb eines Unternehmens geschieht, aus Unternehmensperspektive als interner Kontrollbereich gilt.

\section{Literatur}

Alexander, C. (1999), On the Nature of the Reputational Penalty for Corporate Crime: Evidence, Journal of Law and Economics, Vol XLII, April 1999, S. 489-526.

Bannenberg, B. (2002), Korruption in Deutschland und ihre strafrechtliche Kontrolle. Neuwied.

Battigalli, P. und G. Maggi (2002), Rigidity, Discretion, and the Costs of Writing Contracts, The American Economic Review, S. 798-817

Boers, K., H. Theile und K.-M. Karliczek (2003), Wirtschaft und Strafrecht Wer reguliert wen?, in Karstedt, S. und D. Oberwittler (Hrsg.), Soziologie der Kriminalität, Sonderheft der Kölner Zeitschrift für Soziologie und Sozialpsychologie, 469-493.

Bundeskriminalamt (2001), Jahresbericht Wirtschaftskriminalität, Wiesbaden.

Bussmann, K.-D., 2004. Kriminalprävention durch Business Ethics. Ursachen von Wirtschaftskriminalität und die besondere Bedeutung von Werten in: Zeitschrift für Wirtschafts- und Unternehmensethik, Jahrgang 5, Heft 1, 3550 .

Coase, R.H. (1990), The Firm, the Market, and the law. The University of Chica- 